\section{Rehabilitation in Amyotrophic Lateral Sclerosis: Approaches and Future Prospects}

\section{Marco Orsini ${ }^{1 *}$, Monara Kedma Nunes ${ }^{2}$, Lysnara Lial' ${ }^{2}$, Rayele Moreira $^{2}$, Victor Hugo Bastos ${ }^{2,3}$, André Palma Matta ${ }^{1}$, Mariana Cunha ${ }^{1}$, Beny Smidth ${ }^{4}$, Pedro Ribeiro ${ }^{3}$, Bruna Velasques ${ }^{3}$, Silmar Teixeira ${ }^{5}$, Juliana Bittencount ${ }^{6}$ and Acary Bulle Oliveira $^{7}$}

${ }^{1}$ Department of Neurology, Fluminense Federal University, Rio de Janeiro, Brazil

${ }^{2}$ Brain Mapping Laboratory and Functionality - LAMCEF, Federal University of Piaui, Parnaíba, Brazil

${ }^{3}$ Brain Mapping Laboratory and Sensory-motor Integration, IPUB - Instituto de Psquiatria da UFRJ, Psychiatric Hospital, Rio de Janeiro, Brazil

${ }^{4}$ Department of Neurology, Federal University of São Paulo - UNIFESP, São Paulo, Brazil

${ }^{5}$ Mapping and Brain Plasticity Laboratory - LAPLACE, Federal University of Piaui, Parnaíba, Brazil

${ }^{6}$ Federal University of Rio de Janeiro-UFRJ - Brain Mapping Laboratory and EEG - IPUB, Rio de Janeiro, Brazil

${ }^{7}$ Fluminense Federal University, Rio de Janeiro, Brazil

\section{Introduction}

Amyotrophic Lateral Sclerosis (ALS) is the most common form of motor neuron disease in adults with an estimated incidence of 1-2 cases per 100,000 inhabitants, being the majority of sporadic type and $5-10 \%$ familiar. The progressive sign of weakness, and paralysis of the muscles of the upper and lower limbs, face and trunk observed in individuals with the disease results from the progressive loss of upper motor neurons, the corticospinal tract, bulb and spinal cord [1].

The disease is neurodegenerative in nature and inexorably leads to death within 5-10 years from the onset of symptoms, usually as a consequence of a progressive restrictive respiratory failure with diaphragmatic involvement, the main breathing muscle [2]. Because it is a rare pathology, the diagnosis takes about a year for spine forms and 4-6 months for the bulbar ones, and the later the diagnosis, the shorter survival [3]. There is no healing treatment, therefore, palliative

*Corresponding author: Marco Orsini, Department of Neurology, Fluminense Federal University, Graduate Program in Rehabilitation Sciences, Praça das Nações, 34 - Bonsucesso, Rio de Janeiro - 21041021, Brazil, Tel: +55 (21) 984449832; E-mail: orsinimarco@hotmail.com

Citation: Orsini M, Nunes MK, Lial L, Moreira R, Bastos VH, et al. (2016) Rehabilitation in Amyotrophic Lateral Sclerosis: Approaches and Future Prospects. J Phys Med Rehabil Disabil 2: 008.

Received: November 12, 2015; Accepted: January 28, 2016; Published: February 15, 2016 measures are used in an attempt to ensure longevity of patients, quality of life and reduced costs [2,3].

The main therapeutic pillar in ALS is a trans-disciplinary approach, including health professionals, such as doctors, nurses, speech therapists, physiotherapists, nutritionists, psychologists and occupational therapists [4]. The natural history of the disease with variable evolution speed between patients prevents the protocol of activities/exercise therapy, although it is known that a metabolic overload on an already compromised system should be avoided $[5,6]$ and management of muscle weakness/fatigue should include energy maintenance techniques, changes in lifestyle and regular periods of rest between activities. Proper nutritional support is essential, since about one-third of patients with ALS are found in state of malnutrition [7] and stretching techniques and the use of assistive devices are important to facilitate certain basic and instrumental activities of daily living [6].

Symptoms such as dyspnoea, dysphonia, and dysarthria are crucial for speech intelligibility [8] that decreases as the disease progresses. In neuromuscular affections, the pattern of musculature involves changes in vocal quality, resonance, breathing, articulatory precision, speech rate, prosody and pneumophonoarticulatory coordination. At this stage, the therapist, in order to guarantee the message transmission and a better understanding of the caller, encourages the patient to use resources such as syntactic reduction and the routine use of vocabulary, and graphical support [9].

At a more advanced stage of the disease, the patient needs maximum assistance since he is already being bedridden and dependent on invasive ventilatory support. At this time, passive and stretching exercises are indicated so as to delay the onset of contractures and deformities, or to prevent progression of existing liabilities, the use of invasive or noninvasive mechanical ventilation, tracheal aspiration, a nasogastric tube and psychological support [10].

\section{References}

1. Sanjuán-López $P$, Valiño-López $P$, Ricoy-Gabaldón $\mathrm{J}$, Verea-Hernando $H$ (2014) Amyotrophic lateral sclerosis: impact of pulmonary follow-up and mechanical ventilation on survival. A study of 114 cases. Arch Bronconeumol 50: 509-513.

2. Camacho A, Esteban J, Paradas C (2015) Report by the Spanish Foundation for the Brain on the social impact of amyotrophic lateral sclerosis and other neuromuscular disorders. Neurologia.

3. Jiménez García I, Sala Moya N, Riera Munt M, Herrera Rodríguez MV, Povedano Panadés M, et al. (2015) [The patient's opinion matters: experience in the nutritional care in an ALS multidisciplinary team]. Nutr Hosp 5: 56-66.

4. Majmudar S, Wu J, Paganoni S (2014) Rehabilitation in amyotrophic lateral sclerosis: why it matters. Muscle Nerve 50: 4-13.

5. Pontes RT, Orsini M, de Freitas MRG, de Souza Antonioli R, Nascimento OJM (2010) Alterações da fonação e deglutição na Esclerose Lateral Amiotrófica: Revisão de Literatura. Rev Neurocienc 18: 69-73.

6. Hanisch F, Skudlarek A, Berndt J, Kornhuber ME (2015) Characteristics of pain in amyotrophic lateral sclerosis. Brain Behav 5: 00296.

7. Genton L, Viatte V, Janssens JP, Héritier AC, Pichard C (2011) Nutritional state, energy intakes and energy expenditure of Amyotrophic Lateral Sclerosis (ALS) patients. Clin Nutr 30: 553-559.

8. Gordon PH (2013) Amyotrophic Lateral Sclerosis: An update for 2013 Clinical Features, Pathophysiology, Management and Therapeutic Trials. Aging Dis 4: 295-310. 
Citation: Orsini M, Nunes MK, Lial L, Moreira R, Bastos VH, et al. (2016) Rehabilitation in Amyotrophic Lateral Sclerosis: Approaches and Future Prospects. J Phys Med Rehabil Disabil 2: 008.

- Page 2 of $2 \cdot$

9. Orsini M, Oliveira AB, Nascimento OJ, Reis CH, Leite MA, et al. (2015) Amyotrophic Lateral Sclerosis: New Perpectives and Update. Neurol Int 7: 5885.
10. Orsini M (2012) Reabilitação nas doenças neuromusculares: Abordagem interdisciplinar. Guanabara Koogan, Rio de Janeiro, Brazil. 